\title{
INTERNATIONAL JOURNAL Of RESEARCH
}

\section{संगीत चिकित्सा — एक नवाचार}

लता मेहरा

एम. फिल्. (गायन)

संगीत एवं नृत्य विभाग, कुरुक्षेत्र विश्वविद्यालय, कुरुक्षेत्र

संगीत कला भारतीय संस्कृति की आत्मा है, भारतीय संस्कृति के उद्भव काल से ही संगीत इसका अभिन्न अंग रहा है, वर्तमान समय में व्यस्त, तनावपूर्ण एवं प्रतियोगी सामाजिक परिस्थितियों में संगीत ही एक ऐसी कला है, जो मनुष्य के आहत मन एवं आत्मा को कुछ समय के लिए ही सही सुख प्रदान करती है ।

संगीत की महत्ता को दर्शाते हुए किसी विचारक ने क्या खूब कहा है कि -

\section{"संगीत है ईश्वर की शक्ति हर स्वर में बसे हैं राम, रागी जो सुनाये रागिनी रोगी को मिले आराम ।"}

संगीत मानव के व्यक्तित्व एवं मानसिक विकास में महत्वपूर्ण भूमिका अदा करता है । संगीत मानव के पीड़ित हृदय को संतोष एवं शान्ति तो प्रदान करता ही है, वरन् जीवन में हर्षोल्लास भरकर आगे बढ़ने की प्रेरणा भी देता है ।

संगीत की शक्ति से बीमारियों की चिकित्सा, भूमि की उर्वरा शक्ति को बढ़ाना, पशुओं को नियंत्रित करना, दुधारू पशुओं के दुग्ध में वृद्धि तथा फसलों की पैदावार में वृद्धि आदि अनेक उपलब्धियाँ इतिहास में विद्यमान है ।

समस्त ललित कलाओं में संगीत का स्थान अत्यन्त महत्वपूर्ण है। इससे मनुष्य लौकिक तथा अलौकिक दोनों प्रकार के सुख प्राप्त करता है । संगीत से केवल आनन्दानुभूति ही नहीं होती, ध्वनियाँ मानसिक स्थितियों की भी सूचक होती हैं। साथ ही ये हमारे मनोभावों को भी प्रभावित करती हैं । सर्वप्रथम संगीत के चिकित्सकीय प्रयोग का उल्लेख करते हुए श्री उमेश जोशी ने अपनी पुस्तक "भारतीय संगीत का इतिहास" में लिखा है कि जब कोई बीमार पड़ता था तो लोग दवा नहीं देते थे बल्कि संगीत द्वारा ही उसका उपचार करते थे ।

सुप्रसिद्ध सांगीतिक समीक्षक ऑरसन ली ने "अशार्ट अकाउन्ट ऑफ इंडियन म्यूजिक" (A short account of Indian Music) नामक पुस्तक में लिखा है कि "मुगल दरबार का तानसेन बड़ा चमत्कारी गायक था, उसने दीपक राग गाकर अकबर बादशाह को आश्चर्य में डुबो दिया था, इस राग को गाने पर अग्नि प्रज्जवलित हो उठती थी, इसी प्रकार वीणा वादन से मृगों को बुला लिया करता था ।

बीसवीं सदी में पाश्चात्य देशों ने संगीत चिकित्सा पर व्यापक रूप से अनुसंधान करने शुरू कर दिये तथा इसका उपयोग भी प्रचुर मात्रा में करने लगे ।

फलस्वरूप सन् 1944 ई. में 'मिशीगन' विश्वविद्यालय द्वारा संगीत चिकित्सा का प्रथम पाठ्यक्रम तैयार किया गया । इस पाठ्यक्रम के अनुसार लोगों को शिक्षा दी जाने लगी । इस पाठ्यक्रम को सन् 1946 में सबसे पहले 'कन्यास विश्वविद्यालय' में प्रारम्भ किया गया । अन्य कॉलेजों ने भी शीघ्र ही इसे अपना लिया । सन् 1950 ई. में नेशनल एसोसिएशन फॉर म्यूजिक थेरेपी (N.A.M.T.) नाम से इस कार्य के लिए एक संस्था स्थापित कर दी । इस संस्था के द्वारा संगीत चिकित्सा के प्रमाणपत्र दिये जाने लगे और पंजीकरण आदि होने लगे।

इसके अतिरिक्त भी विदेशों में संगीत चिकित्सा यथा-

The foot taps in time, the head nods or a finger moves rhythmically. We may assume that muscular tension and relaxation are present as a response to music, even where they do not make themselves felt as overt movement. 


\section{INTERNATIONAL JOURNAL Of RESEARCH -GRANTHAALAYAH \\ A knowledge Repository}

Art

अर्थात् पैर समय पर थपकते हैं। सिर का हिलना या फिर उंगलियों का लय में हिलना। हम यह मान सकते हैं कि पेशीय तनाव तथा शिथिलन संगीत के जवाब में दिखता है। कभी-कभी तो वे इस प्रकट हुई चाल का अनुभव ही नहीं करते ।

संगीत एवं हंसी आदि से मानसिक तनाव भी कम होता है तथा शरीर भी निरोगी रहता है, इसी तरह गुस्सा, तनाव, थकान आदि शारीरिक तथा मानसिक विकारों से शरीर की प्रतिरोधक शक्ति भी कम होती है । संगीत में इतनी ऊर्जा होती है कि जीवन से हार चुके व्यक्ति में भी संगीत नई स्फूर्ति को जगाता है, तन-मन को शान्ति प्रदान कर जीवन जीने की प्रेरणा देता है संगीत ।

चूंकि संगीत, ध्यान आदि विधायें मनुष्य के मस्तिष्क को विचारहीन करके उसका ध्यान केन्द्रित ही नहीं करती बल्कि जब संगीत सुना जाता है तो पसंदीदा संगीत के श्रवण से मानसिक शान्ति एवं आनन्द की अनुभूति भी होती है । इस समय मस्तिष्क तो प्रभावित होता ही है, साथ ही साथ नलिकाविहीन ग्रन्थियाँ आदि भी प्रभाहित होती हैं । जिस वजह से इनके द्वारा स्त्रावित हार्मोन्स में होने वाले परिवर्तनों के कारण सम्पूर्ण शरीर भी एवं समस्त अंग आदि भी प्रभावित होते है । तनावमुक्त स्थिति में ही शरीर के हार्मोन्स सुचारू रूप से कार्य करते हैं। कहने का तात्पर्य यह है कि संगीत सुनकर या ध्यानादि क्रियाओं के द्वारा अपने आपको प्रसन्न एवं तनावरहित रखकर हम अपने आपको स्वस्थ रख सकते हैं । साथ ही साथ कई रोगों से छुटकारा भी पा सकते हैं । इसी बात की पुष्टि करते हुए श्री कुमार अंचल ने अपने लेख "विधाता की अद्भुत कृति है हमारा दिमाग" में लिखा है कि - 'युवक कर्णप्रिय संगीत सुनकर अपने सामान्य से अधिक रक्तचाप को ठीक कर सकते हैं, अधिक पसीना निकलने तथा श्वास में होने वाली तकलीफ को भी ठीक कर सकते हैं ।'

सांगीतिक चिकित्सा के साधन - संगीत का उपयोग चिकित्सा के रूप में अतिप्राचीन काल से ही किया जा रहा है । संगीत के इतिहास का अध्ययन करने पर हमें यह ज्ञात होता है कि संगीत में चिन्हित चिकित्सकीय एवं उपचारीय प्रभाव को मनुष्य ने काफी पूर्व में ही जान लिया था तथा संगीत का उपयोग रोगों के उपचार हेतु करना आरम्भ कर दिया था । सांगीतिक इतिहास का अध्ययन करने पर द्रविड़ों द्वारा संगीत चिकित्सा किये जाने का वर्णन हमें प्राप्त होता है । इसके पश्चात् हमारे प्राचीन शास्त्रियों ऋषियों आदि ने शब्द एवं स्वर के प्रभाव को किस गहराई तक समझ लिया था, इसका उदाहरण हमें वैदिक काल में रचित हमारे चारों वेदों के रूप में प्राप्त होता है। जिसमें से सामवेद को केवल गायन के लिए ही निर्मित किया गया तथा पूर्णरूपेण गेय बनाया ।

सामवेद के उपवेद गंधर्ववेद में तो स्वरों के प्रभाव आदि विषयों पर विशिष्ट जानकारी लिखी गई, लेकिन यह दुर्भाग्य है कि आज उसकी एक भी प्रति आज उपलब्ध नहीं है । इसके पश्चात् इन विशिष्ट स्वरों के गठन से मंत्रों का निर्माण किया गया तथा इस प्रकार मंत्र चिकित्सा के रूप में एक नई चिकित्सा पद्धति का प्रारम्भ हुआ, जोकि वास्तव में संगीत चिकित्सा का ही एक रूप था। उसके द्वारा चिकित्सकीय प्रभाव उत्पन्न करना, मंत्र चिकित्सा को उस समय उतना ही महत्व दिया जाता था, जितना कि अन्य चिकित्सा पद्धतियों को योग विशिष्ट में मंत्र की चिकित्सा शक्ति पर प्रकाश डालते हुए लिखा है-

"यथा विरेक कुर्वन्ति हरीतक्यः स्वभावतः ।

भावनावशतः कार्य तथा यरलवादयः ।।

अर्थात- जैसे हर्र खाने से पाचन संस्थान में तीव्र गति होती है और दस्त लग जाते हैं, उसी प्रकार दृढ़ भावना से यरलव आदि मंत्रों के अक्षर शरीर पर असर डालते हैं ।

"म्यूजिक फॉर 'हेल्थ' में चार हजार पूर्व संगीत का चिकित्सा के रूप में उपयोग के विषय में लिखा है कि -

\section{'Four thousand years ago the friest doctors of Egypt had a fovourite Incantation in music which purported To have a fovourable inteuence the fertility of women}

अर्थात् चार हजार वर्ष पूर्व मिश्र के पादरी चिकित्सक के पास संगीत का एक पसंदीदा मंत्र था, जिसका कि महिलाओं की प्रजनन क्षमता पर अनुकूल प्रभाव बताया जाता था । 


\section{INTERNATIONAL JOURNAL Of RESEARCH -GRANTHAALAYAH \\ A knowledge Repository}

Art

यदि हम गायन तथा वादन के प्रभाव की विवेचना करें तो हम पाते हैं कि जहाँ गायन अपने शब्दों और स्वरों के कारण जल्द रस उत्पत्ति कर देता हैं, वहीं गायन में गीत तथा राग इन दोनों के रसों में विभिन्नता होने की वजह से इसका प्रभाव हमेशा एक सा नहीं हो पाता, जिसके फलस्वरूप चिकित्सा में कठिनाई उत्पन्न हो सकती है ।

इसकी अपेक्षा तन्त्री वाद्यों या फिर अन्य किसी भी वाद्य पर बजाये जाने वाले राग आदि का रस एवं प्रभाव हमेशा एक ही रहता है । इसलिए तन्त्री वाद्यों को संगीत चिकित्सा के लिए ज्यादा उपयुक्त एवं प्रभावशाली माना गया है ।

डॉ. जेक्सन पाल ने अपनी पुस्तक "संगीत चिकित्सा" में संगीत चिकित्सा हेतु वाद्यों के चयन के विषय में लिखा है कि यद्यपि अपने-अपने स्थान पर सभी अच्छे हैं, परन्तु सितार और सारंगी इन दो का प्रधान स्थान है और इन दोनों में भी सितार मुख्य है ।

इन दोनों वाद्यों में भी सितार को ज्यादा प्रभावी एवं महत्वपूर्ण बताते हुए डॉ. जेक्सन पाल ने लिखा है कि, 'इसमें पखावज या मृदंग की आवश्यकता नहीं , उंगली की गति ताल के अनुरूप ही चलती है, दूसरे एक बंद पर उंगली रखकर एक ही स्वर पर जितना चाहे कंप दे सकते हैं और तीसरे जिस स्वर को चाहे बजाते हुए जोड़े और पपीहे द्वारा उसमें मधुरता ला सकते हैं ।

संगीत की तीनों विधाओं में शरीर को स्वस्थ एवं हष्ट-पुष्ट करने की शक्ति है । इसकी तुलना यदि हम साधारण व्यायाम से करें तो भी इसकी श्रेष्ठता हमें अलग ही दिखाई देती है । चूंकि दोनों में जो मूलभूत अन्तर है वह यह है कि शारीरिक व्यायाम के समय अंगों का कभी तीव्र गति से तो कभी मन्द गति से व्यायाम होता है एवं इस तरह की उत्तेजना शरीर के लिए हानिकारक भी हो सकती है । इसके विपरीत संगीत से शारीरिक अंगों का व्यायाम हमेशा एक सा होता है तथा इन अंगों पर पड़ने वाला दबाव भी हमेशा एक सा होने की वजह से उन पर किसी भी तरह का हानिकारक प्रभाव नहीं पड़ता ।

इसके अतिरिक्त विभिन्न रोगों के उपचार हेतु चिकित्सकों ने संगीत का उपयोग किया है । किसी भी बीमार व्यक्ति के लिए नींद एक आवश्यक तत्व है । संगीत चिकित्सा द्वारा सर्वप्रथम रोगी को नींद दिलाने की कोशिश की जाती है।

जाने माने संगीतज्ञ तथा पं. विष्णु दिगम्बर के शिष्य प्रोफेसर व्ही.ए. कषालकर ने रोगी को नींद लाने के लिए संगीत की सहायता लेने का सुझाव दिया । आखिरकार इस प्रकार की स्वीकृति दे दी गई तथा यह पूर्णतः सफल रहा, रोगी के बिस्तर के पास मंद्र तथा मध्य सप्तक में आलाप किये गये, जिसके कारण रोगी घंटों गहरी नींद सोता रहा ।

केवल इतना ही नहीं कि संगीत कम विकसित मस्तिष्क या कम बौद्धिक क्षमता वाले मनुष्यों पर ही अपना प्रभाव डालता है, अपितु एक साधारण मनुष्य को भी यदि संगीत सुनाया जाये तो उसकी बौद्धिक क्षमता और भी अधिक बढ़ जाती है ।

संगीत चिकित्सा पर अनुसंधान कर रहे वैज्ञानिकों एवं चिकित्सकों ने संगीत को दर्द निवारक के रूप में काफी उपयोगी पाया है, चूंकि आजकल की जा रही खोजों से यह ज्ञात हुआ है कि दर्द की कमी तथा तीव्रता काफी कुछ मनुष्य की उसके प्रति संवेदनशीलता पर भी निर्भर करती है ।

मानसिक रोगों और विकारों से ग्रस्त रोगियों पर संगीत के इस प्रभाव को देखते हुए मानसिक चिकित्सकीय संगीत को मानसिक रोगों की चिकित्सा हेतु अति उपयोगी मान रहे हैं तथा इस हेतु इसका उपयोग भी कर रहे हैं ।

संगीत चिकित्सा पर अनुसंधानरत तथा संगीत का चिकित्सा के रूप में प्रयोग कर रहे कार्ला महाराष्ट्र के निवासी चिकित्सक डॉ。 बालाजी ताम्बे ने अपने प्रयोग में कई भारतीय शासत्रीय संगीत के रागों को कई विशेष रोग के लिए लाभकारी पाया है । डॉ॰ ताम्बे के अनुसार- राग भूपाली तथा तोड़ी उच्च रक्तचाप के रोगियों को अधिक आराम देते हैं । जबकि राग मालकोंस तथा आसावरी निम्न रक्तचाप के रोगियों को पूर्व अवस्था में पहुंचाने में सहायता करते हैं । राग भैरवी मानसिक शिथिलता लाता है ।

एनसीफेलोग्राम के माध्यम से विभिन्न प्रकार के संगीत का प्रयोग विभिन्न रोगियों के उपचार के लिए किया जाता है । विशेष रूप से संगीत के द्वारा मानसिक व्याधियों, उदासीनता, अवसाद, उच्च रक्तचाप, निम्न रक्तचाप, जोड़ों का दर्द, पाचनक्रिया सम्बन्धी रोग मनोरोग तथा नशा आदि समस्याओं का समाधान किया जाता है । 


\section{INTERNATIONAL JOURNAL Of RESEARCH -GRANTHAALAYAH \\ A knowledge Repository} Art

जैसे स्मरण शक्ति के लिए शिवरंजनी, तनावमुक्ति के लिए तोड़ी, मालकौंस, आसावरी, बिहाग, मुल्तानी तथा उच्च तथा निम्न रक्तचाप के लिए आनन्दी एवं मलेरिया, टायफायड, कब्ज तथा हिस्टीरिया के लिए हिंडोल, मारवा, पूरिया तथा रामकली आदि रागों का प्रयोग किया जाता है ।

मन को एकाग्र करने का एक उचित साधन संगीत ही है । संगीत-योग द्वारा व्यक्ति सहज ही आनन्द की प्राप्ति करता है । तनाव के कारण कई व्याधियाँ होती हैं । जैसे- पेट का अल्सर, गुर्दे का रोग, थायरायड, हृदय रोग, डायबिटीज, उच्च व निम्न रक्तचाप, इन भयावह स्थितियों से निपटने के लिए चिकित्सक तथा मनोचिकित्सक प्रयोगों में रत हैं ।

इस तरह हम संगीत चिकित्सा पद्धति की तुलना अन्य चिकित्सा पद्धति से करें तो हमें इस चिकित्सा पद्धति में कुछ ऐसी विशेषताएँ दिखाई देती हैं, जो इसकी श्रेष्ठता को सिद्ध करती हैं ।

संगीत चिकित्सा की भी अपनी विशेषता तथा सीमायें है । आज हमारे भारतवर्ष में यह पद्धति कम प्रचलित होने के साथ-साथ इस पर शोधकार्य भी कम ही किये जा रहे हैं एवं इस चिकित्सा हेतु साहित्य का भी अभाव है । किन्तु इस पर यदि शोधकार्य और प्रयोग किये जाते रहे तो इससे छिपी कई और विशेषताएँ हमें प्राप्त हो सकेंगी तथा रोगियों के रोग निवारण हेतु एक मनपसन्द, दुष्परिणामरहित सफल चिकित्सा पद्धति प्राप्त हो सकेगी ।

सन्दर्भ -

1 'संगीत चिकित्सा' - डॉ॰ सतीश वर्मा भारतीय, शास्त्रीय संगीत मनोवैज्ञानिक आयाम - डॉ॰ साहित्य कुमार नाहर 